\title{
Comparative study on the diagnostic value of intravenous/ peritumoral injection of indocyanine green for metastatic lymph node location in patients with head and neck squamous cell carcinoma (HNSCC)
}

\author{
Chengwan Xia $^{1 \# \wedge}$, Qunzhi Zhou ${ }^{1 \#}$, Qian Zhang ${ }^{1}$, Shiqi $\mathrm{Hu}^{1}$, Elisa Meacci ${ }^{2,3}$, Yosuke Matsuura ${ }^{4}$, \\ Marion Durand ${ }^{5}$, Qingang $\mathrm{Hu}^{1}$, Huiming Cai ${ }^{6}$, Yuxin Wang ${ }^{1}$ \\ ${ }^{1}$ Department of Oral and Maxillofacial Surgery, Nanjing Stomatological Hospital, Medical School of Nanjing University, Nanjing, China; \\ ${ }^{2}$ Department of General Thoracic Surgery, Fondazione Policlinico Universitario A. Gemelli IRCCS, Rome, Italy; ${ }^{3}$ Università Cattolica del Sacro \\ Cuore, Rome, Italy; ${ }^{4}$ Department of Thoracic Surgical Oncology, The Cancer Institute Hospital, Japanese Foundation for Cancer Research, Tokyo, \\ Japan; ${ }^{5}$ Ramsay Santé, Thoracic Unit, Hôpital Privé d'Antony, Antony, France; ${ }^{6}$ Department of Biomedical Engineering, College of Engineering and \\ Applied Sciences, Nanjing University, Nanjing, China \\ Contributions: (I) Conception and design: C Xia, Y Wang, Q Hu, H Cai; (II) Administrative support: Q Hu; (III) Provision of study materials or \\ patients: Y Wang; (IV) Collection and assembly of data: C Xia, Y Wang; (V) Data analysis and interpretation: C Xia, Q Zhou, Q Zhang, S Hu; (VI) \\ Manuscript writing: All authors; (VII) Final approval of manuscript: All authors. \\ \#These authors contributed equally to this work. \\ Correspondence to: Yuxin Wang. Department of Oral and Maxillofacial Surgery, Nanjing Stomatological Hospital, Medical School of Nanjing \\ University, Nanjing, China. Email: wangyuxin0212@126.com; Qingang Hu. Department of Oral and Maxillofacial Surgery, Nanjing Stomatological \\ Hospital, Medical School of Nanjing University, Nanjing, China. Email: qghu@nju.edu.cn; Huiming Cai. Department of Biomedical Engineering, \\ College of Engineering and Applied Sciences, Nanjing University, Nanjing, China. Email: chm9166@aliyun.com.
}

Background: Accurate assessment of regional lymph node (LN) status is essential for the treatment of head and neck squamous cell carcinoma (HNSCC) patients. In this study, we aimed to compare the difference between intravenous injection of indocyanine green (ICG) and peritumoral injection of ICG in the location of metastatic LNs.

Methods: Twenty-nine patients were enrolled in this study with 13 patients receiving intravenous injection of ICG and 16 patients receiving peritumoral injection of ICG. During the surgery, the fluorescence-positive LNs in vivo were sent to undergo frozen section after fluorescence intensity was recorded. After the cervical LN dissection, all LNs were sorted by region, and the fluorescence intensity was recorded before the LNs were sent for paraffin section.

Results: During the surgery, both intravenous or peritumoral injections with near-infrared (NIR) fluorescence imaging of ICG had their respective pros and cons in vivo, with the sensitivity and specificity being $62.5 \% / 75 \%$ and $98.1 \% / 89.1 \%$ respectively. After the surgery, both methods could reduce the pathological workload by preselecting the LNs at-risk in the premise of accurate assessing the cervical LN stage. However, intravenous ICG administration was more valuable in determining all types of LN status according to the fluorescence intensity [area under the curve (AUC): 0.91 vs. $0.78, \mathrm{P}<0.001$ ].

Conclusions: With the assistance of NIR fluorescence imaging using ICG, both administration methods could reduce the postoperative complication and the pathological workload, whereas the intravenous mode of ICG administration is superior in application value.

\footnotetext{
^ ORCID: 0000-0003-3937-2605.
} 
Keywords: Head and neck squamous cell carcinoma (HNSCC); indocyanine green (ICG); metastatic lymph node; fluorescence intensity quantification; pathological examination

Submitted Oct 19, 2020. Accepted for publication Feb 26, 2021.

doi: $10.21037 / \mathrm{atm}-21-392$

View this article at: http://dx.doi.org/10.21037/atm-21-392

\section{Introduction}

For solid tumors, such as those found in head and neck squamous cell carcinoma (HNSCC), regional lymph node metastasis has proven to be an important independent factor affecting the treatment and the prognosis of patients $(1,2)$. According to the 2020 National Comprehensive Cancer Network (NCCN) guidelines, for HNSCC patients with LN metastasis, radical neck dissection and necessary postoperative chemoradiotherapy are required, while for those HNSCC patients without LN metastasis, selective neck dissection or no cervical treatment is considered more appropriate. However, missed or faulty diagnosis often occurs with conventional diagnostic methods such as computed tomography (CT), magnetic resonance imaging (MRI), ultrasound, and positron emission tomography-CT (PET-CT) (3-5). In order to avoid local recurrence, surgeons often choose to perform neck dissection because HNSCC tends to metastasize to regional LNs at the early stage. In fact, one review of the postoperative pathology of HNSCC patients revealed that only $30 \%$ actually suffered LN metastasis $(\mathrm{pN}+)(6)$. This means the remaining $70 \%$ of patients ( $\mathrm{pN} 0)$ were subjected to unnecessary postoperative complications related to neck dissection, including skin numbness and shoulder morbidity (7). There is therefore an urgent need to develop a minimally invasive/non-invasive method to accurately locate metastatic LNs, especially in the preoperative or peri-operative period. At present, pathological examination remains the gold standard for determining LN status. In pre-operation, fine needle aspiration biopsy (FNAB) was found to perform adequately in reducing the surgical trauma and pathological workload (8). This technique has shown usefulness in determining whether a tumor is a benign or malignant. However, the high false-negative $(\sim 50 \%)$ rates in detecting metastatic LNs and the risk of tumor cell implantation dissemination have hindered its clinical application (9). The sentinel lymph node (SLN) is the first LN in the lymphatic basin to be affected by metastatic tumor cells from the primary tumor (10). Based on this theory, SLN biopsy (SLNB) is another technique which evaluates $\mathrm{N}$ stage by detecting tumor cells in the SLN during operation (11). SLNB has been widely used in the treatment of breast carcinoma, malignant melanoma, HNSCC, and other tumors $(12,13)$. The accurate location of the SLN is the crux of this technique's success. In previous studies, researchers have often chosen peritumoral injection of tracer, such as methylene blue or nuclide, to locate SLNs, but each of these methods has its respective shortcoming, such as low sensitivity/specificity, radioactive contamination, and the shine-through phenomenon (13-18).

In recent years, with the improvement of fluorescent agents and imaging equipment, near-infrared (NIR) fluorescence imaging technology has been widely used in the biological and medical fields (19-22). In oncology, NIR fluorescence imaging has already been applied to the delineation of tumor boundary, the identification of residual tumor cells, and SLN mapping. Indocyanine green (ICG) is one of the NIR fluorescence dyes approved by the U.S. Food and Drug Administration (FDA). We have already confirmed the feasibility of delineating the HNSCC tumor boundary and monitoring the residual tumor cells by NIR fluorescence imaging using ICG. Interestingly, Nishio et al. have recently proven the value of molecular imaging with panitumumab-IRDye $800 \mathrm{CW}$ in preselecting at-risk LNs (23). More surprisingly, we also have found that ICG fluorescence after intravenous injection was present in metastatic LNs, but not in non-metastatic LNs. Even so, the relationship between fluorescence-positive LNs and metastatic LNs still needs further clarification. Moreover, the difference between the intravenous injection of ICG and the peritumoral injection of ICG for locating metastatic LNs requires further exploration.

In this study, we aimed to compare the diagnostic value of intravenous/peritumoral injection of ICG for metastatic lymph node location in patients with HNSCC. Both peritumoral injection of ICG and intravenous injection of ICG were performed in patients with HNSCC. Before cervical LNs were dissected, the cervical region was 
monitored by an NIR fluorescence imaging system, and the fluorescence-positive LNs were sent to undergo frozen section intraoperatively, and paraffin section postoperatively. The specificity and sensitivity were recorded to compare the diagnostic value of an intravenous injection of ICG versus that of a peritumoral injection of ICG for locating metastatic LNs in patients with HNSCC.

We present the following article/case in accordance with the STARD reporting checklist (available at http://dx.doi. org/10.21037/atm-21-392).

\section{Methods}

\section{Patients enrolled in this study}

The study was conducted in accordance with the Declaration of Helsinki (as revised in 2013). This study was approved by the medical ethics committee of the Institute Affiliated Stomatology Hospital, Medical School of Nanjing University (2017NL-001). Written informed consent was obtained from all patients. Adult patients with biopsy-proven HNSCC and clinical examination-proven cN0 status scheduled to undergo standard-of-care surgery with curative intent were included in this study. TNM stage was classified using the American Joint Committee on cancer (AJCC) seventh edition criteria (24). Exclusion criteria included prior head and neck chemoradiotherapy, prior allergic reaction to ICG, hyperthyroidism and iodine allergic reaction, pregnant/prepregnant status, and severe dysfunction of the visceral organ. From January 2018 to December 2020, 29 patients were enrolled in this study. In all, 16 patients received intravenous injection of ICG while the other 13 patients received peritumoral injection of ICG. All decisions on operation schemes were made after multidisciplinary discussion.

\section{Workflow of peritumoral injection of ICG}

All patients were treated under general anesthesia. Before the injection of the ICG solution, the skin of the neck was incised, and the subplatysmal flap and sternocleidomastoid were raised carefully in order to expose the lymphatic tissue fully. Then, $0.1 \mathrm{~mL} \mathrm{ICG}(5 \mathrm{mg} / \mathrm{mL}, 25 \mathrm{mg}$ of ICG was diluted into $5 \mathrm{~mL}$ of distilled water, Yichuang Pharmaceutical Co., Ltd, Dandong China,) was injected at four or five positions around the tumor (25). The fluorescence image was captured by a NIF imaging-guided instrument (REAL-IGS, Nuoyuan Medical Devices Co.,
Ltd, Nanjing, China) and the fluorescence-positive LNs were removed for rapid intraoperative frozen section after the fluorescence intensities were measured by a handheld NIR fluorescence spectrometer (MaYa 2000 Pro, Ocean Optics, Dunedin, FL, USA). Finally, primary tumor resection and LN dissection were carried out according to the preoperative schemes. All LNs were sorted for routine pathological examination after surgery. Data on location, number, fluorescence intensity, histopathological status of fluorescence-positive LNs, and other LNs were recorded.

\section{Workflow of intravenous injection of ICG}

Twelve hours before surgery, $0.75 \mathrm{mg} / \mathrm{kg}$ of ICG solution (ICG was diluted into $30 \mathrm{~mL}$ of distilled water) was injected through the antecubital vein via an intravenous pump for over 30 minutes (26). During surgery, the fluorescence image of lymphatic tissues in the neck region was captured by a NIF imaging-guided instrument after the subplatysmal flap and sternocleidomastoid were raised carefully. Then, the fluorescence-positive LNs were removed to detect the fluorescence intensity before being sent for intraoperative frozen section. Finally, primary tumor resection and LN dissection was carried out according to the preoperative schemes. Data on location, number, fluorescence intensity, and pathological status of fluorescence-positive LNs, and other LNs were recorded.

\section{Pathological examination}

During the surgery, after embedding with tissue freezing medium (OCT, Neg-50, Thermo Scientific Richard-Allan Scientific, USA) $4 \mu \mathrm{m}$ thick sections were sliced from the LNs and stained with hematoxylin and eosin (HE). After the surgery, all LNs were sent to the pathological department to undergo general pathological examination including HE staining and immunohistochemical staining of the $4 \mu \mathrm{m}$ thick paraffin sections (Figure 1). The slides were diagnosed by two senior pathologists, who were blinded to the fluorescence status, to confirm the presence or absence of tumor cells in the LNs.

\section{Statistical analysis}

Statistical analysis was performed using SPSS statistical software (version 23.0; IBM, NY, USA) and GraphPad Prism (Version 6.0, GraphPad Software, La Jolla, CA, USA). For the fluorescence intensity comparison between 

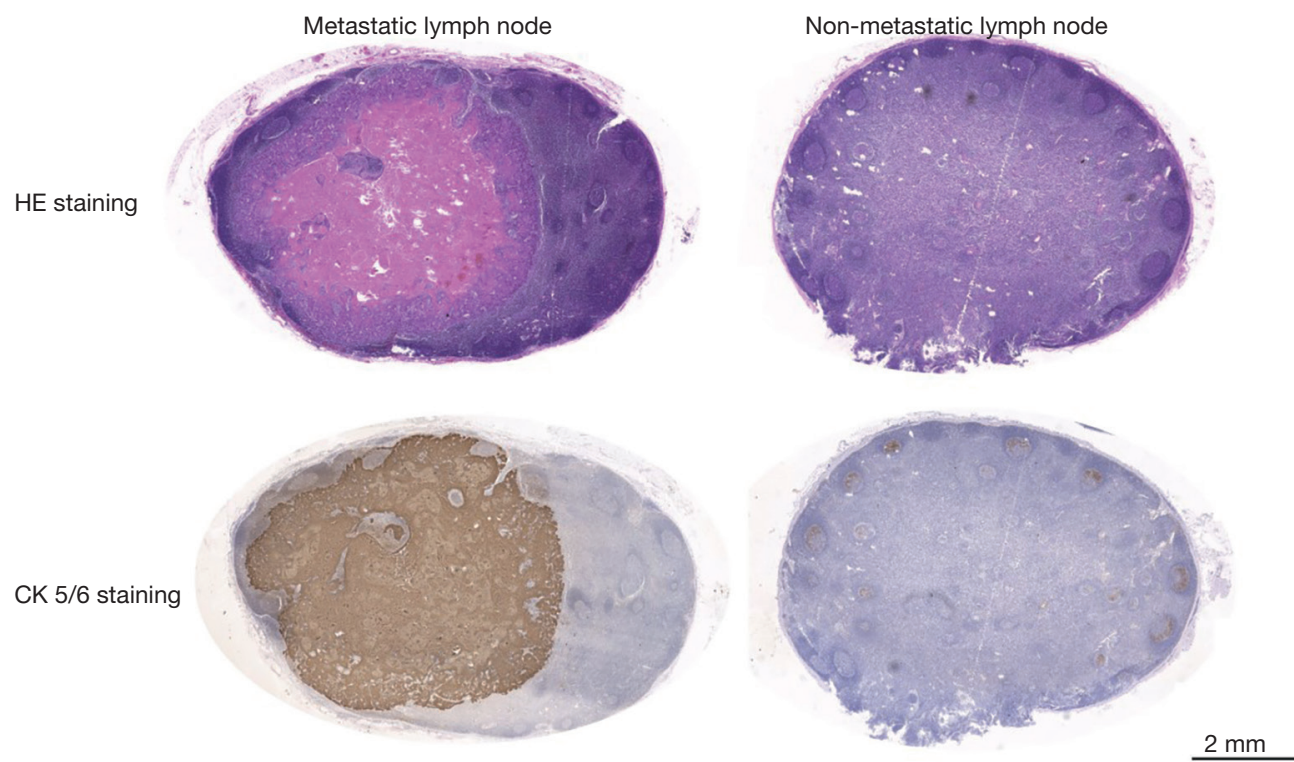

Figure 1 Pathological images (HE staining and CK5/6 immunohistochemical staining) of metastatic LNs and non-metastatic LNs. LNs, lymph nodes; HE, hematoxylin and eosin.

metastatic LNs and non-metastatic LNs, data are presented as mean $\pm \mathrm{SD}$ (range); the differences were compared by a two-sided Student's $t$-test. For the fluorescence intensity comparison between LNs from the intravenous injection of ICG groups and peritumoral injection of ICG groups, data are presented as mean $\pm \mathrm{SD}$, and the differences were compared by a two-sided Student's $t$-test. Sensitivity and specificity for the identification of metastatic LNs by intravenous injection of ICG and peritumoral injection of ICG were calculated by receiver operating characteristic (ROC) curve. Threshold values were determined based on Youden's index, and the likelihood ratio (LR) was defined as follows: $\mathrm{LR}=$ sensitivity/(1-specificity). The area under the ROC curve (AUC) comparison between the intravenous injection of ICG groups and the peritumoral injection of ICG groups was compared by a two-sided Z-test. A P value $\leq 0.05$ was deemed significant, and is indicated by asterisks in the figures.

\section{Results}

\section{Study design}

A single-center, non-randomized study was performed in 29 patients with biopsy-proven HNSCC. All patients received at least one clinical examination before surgery and were diagnosed with $\mathrm{cN} 0$ status; 13 patients received intravenous injection of ICG while the other 16 patients received peritumoral injection of ICG. On the day of surgery intraoperative, NIR fluorescence imaging was performed to access the cervical LNs in vivo during surgery. The fluorescence-positive LNs were marked by methylene blue and sent to undergo frozen section. After surgical resection, the LNs were sorted by region, and fluorescence intensity was detected by a hand-held NIR fluorescence spectrometer. All LNs were sent to undergo paraffin section post-operation. The detailed workflow is illustrated in Figure 2.

\section{Patient characteristics}

Table 1 shows the characteristics of the 13 patients who received intravenous injection of ICG and 16 patients who received peritumoral injection of ICG. For the former, the mean age of patients was 65.1 (range, 50-71). Among them, $5(38.5 \%)$ patients had tumors in the tongue, $6(46.2 \%)$ patients had tumors in the buccal mucosa, $1(7.7 \%)$ patient had tumors in the palatal mucosa, and $1(7.7 \%)$ patient had tumors in the lower lip. Furthermore, $12(92.3 \%)$ patients underwent unilateral neck dissection, while $1(7.7 \%)$ patient underwent bilateral neck dissection. Following pathological examination, 4 (3.8\%) patients had regional LN metastasis, and 5 metastatic LNs were ultimately identified. For the latter, the mean age of 


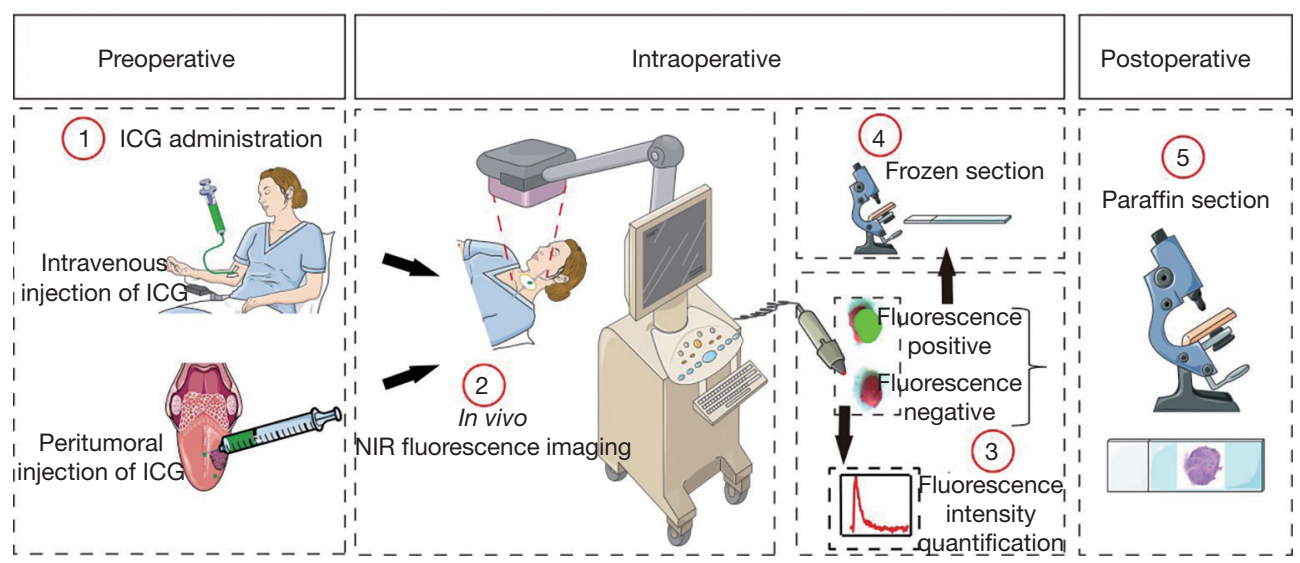

Figure 2 Workflow of NIR fluorescence imaging for patients with HNSCC. Patients were enrolled in two cohorts: intravenous injection of ICG and peritumoral injection of ICG. After ICG was given, NIR fluorescence imaging was performed in the cervical region, and fluorescence-positive LNs were sent for frozen section intraoperatively. After the fluorescence intensity was recorded, all LNs were sent to undergo paraffin section. NIR, near-infrared; HNSCC, head and neck squamous cell carcinoma; ICG, indocyanine green; LNs, lymph nodes.

Table 1 Characteristics of patients who received intravenous/peritumoral injection of ICG

\begin{tabular}{|c|c|c|}
\hline Characteristic & Intravenous injection of ICG, N (\%) & Peritumoral injection of ICG, N (\%) \\
\hline Number of patients & 13 & 16 \\
\hline Gender, male & $6(46.2)$ & $11(68.8)$ \\
\hline \multicolumn{3}{|l|}{ Primary tumor site } \\
\hline Tongue & $5(38.5)$ & $9(56.3)$ \\
\hline Palatal mucosa & $1(7.7)$ & $1(6.3)$ \\
\hline Lower lip & $1(7.7)$ & \\
\hline Mandible & & $1(6.3)$ \\
\hline Pharynx & & $1(6.3)$ \\
\hline T3 & 0 & 5 \\
\hline $\mathrm{T} 4$ & 4 & 1 \\
\hline \multicolumn{3}{|l|}{ Pathological N-stage } \\
\hline No & $9(69.2)$ & $13(81.3)$ \\
\hline N1 & $3(23.1)$ & $1(6.3)$ \\
\hline $\mathrm{N} 2$ & $1(7.7)$ & $2(12.5)$ \\
\hline \multicolumn{3}{|l|}{ Surgical procedure } \\
\hline Unilateral neck dissection & $12(92.3)$ & $15(93.8)$ \\
\hline
\end{tabular}

ICG, indocyanine green. 


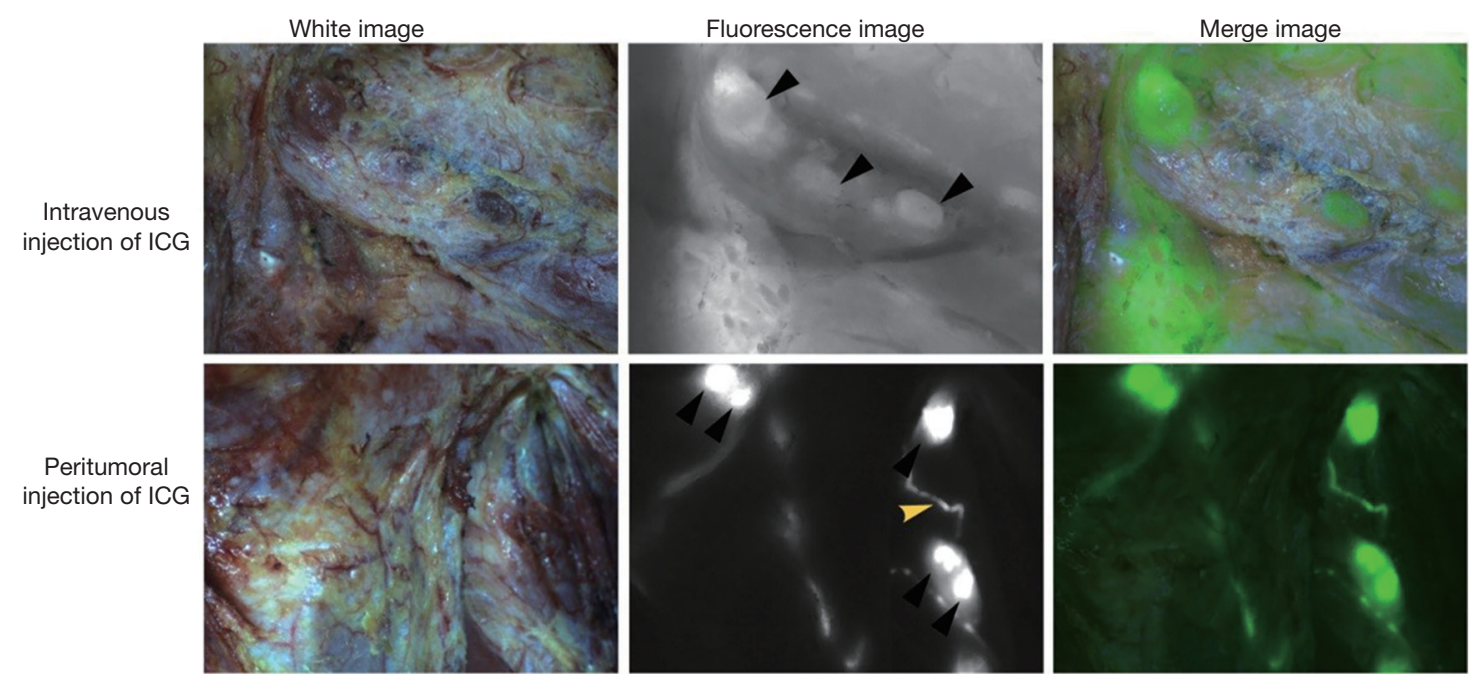

Figure 3 Intraoperative NIR fluorescence imaging for HNSCC patients. The black triangle indicates the fluorescence-positive LNs. The yellow triangle indicates the lymphatic duct. Regional LNs were easier to distinguish after peritumoral injection of ICG than after intravenous injection of ICG. NIR, near-infrared; HNSCC, head and neck squamous cell carcinoma; LNs, lymph nodes; ICG, indocyanine green.

patients was 65.7 (range, 54-78). Among them, 9 (56.3\%) patients had tumors in the tongue, $4(25 \%)$ patients had tumors in the buccal mucosa, 1 (6.3\%) patient had tumors in the palatal mucosa, $1(6.3 \%)$ patient had tumors in the mandible, and 1 (6.3\%) patient had tumors in the pharynx. Furthermore, 15 patients (93.8\%) underwent unilateral neck dissection, and 1 patient (6.2\%) underwent a bilateral neck dissection. Following pathological examination, 3 patients had regional LN metastasis, and 6 metastatic LNs were ultimately identified.

No adverse reactions or complications related to the ICG injection occurred during the study period.

\section{NIR fluorescence imaging for HNSCC patients who received intravenous injection of ICG}

To evaluate the sensitivity and specificity of NIR fluorescence imaging using intravenous injection of ICG for the identification of metastatic LNs, we performed NIR fluorescence imaging to assess cervical LNs. A total of 11 fluorescence-positive LNs were found in 6 (46.2\%) patients in vivo (Figure 3); within the group of 11 fluorescence-positive LNs, 5 were identified as metastatic LNs by intraoperative frozen section in 4 (66.6\%) patients. After LN dissection, cervical LNs were sorted by region. A total number of 332 (range, 11-54 per patient) LNs were harvested (Figure 4), 29 of which (8.7\%) were located in level Ia, 46 of which (13.9\%) were located in level Ib, 93 of which $(28.0 \%)$ were located in level II, 106 of which $(31.9 \%)$ were located in level III, and 58 of which $(17.5 \%)$ were located in level IV. Following postoperative paraffin section, 8 (2.4\%) metastatic LNs were identified, 5 of which $(62.5 \%)$ were fluorescence-positive, and 3 of which $(37.5 \%)$ were fluorescence-negative. With $\mathrm{LN}$ as the unit, the sensitivity and specificity were $62.5 \%$ and $98.1 \%$ respectively, the positive predictive value (PPV) and negative predicative value (NPV) were $45.5 \%$ and $99.1 \%$ respectively, and the accuracy was $97.3 \%$ (Table 2). With the patient as the unit, the accuracy of NIR fluorescence imaging using intravenous injection of ICG combined with intraoperative frozen section was $92.3 \%$.

\section{NIR fluorescence imaging for HNSCC patients who received peritumoral injection of ICG}

NIR fluorescence imaging was also performed to map suspected metastatic LNs in patients who received a peritumoral injection of ICG in vivo at the time of administration and 15 minutes after. A total of 47 (range, 1-6) LNs were found in 16 patients $(100 \%)$ in vivo (Figure 3); within the group of 47 fluorescence-positive LNs, $6(12.8 \%)$ were identified as metastatic LNs by intraoperative frozen section in $3(18.8 \%)$ patients. After LN dissection, a total number of 384 (range, 16-34 per 


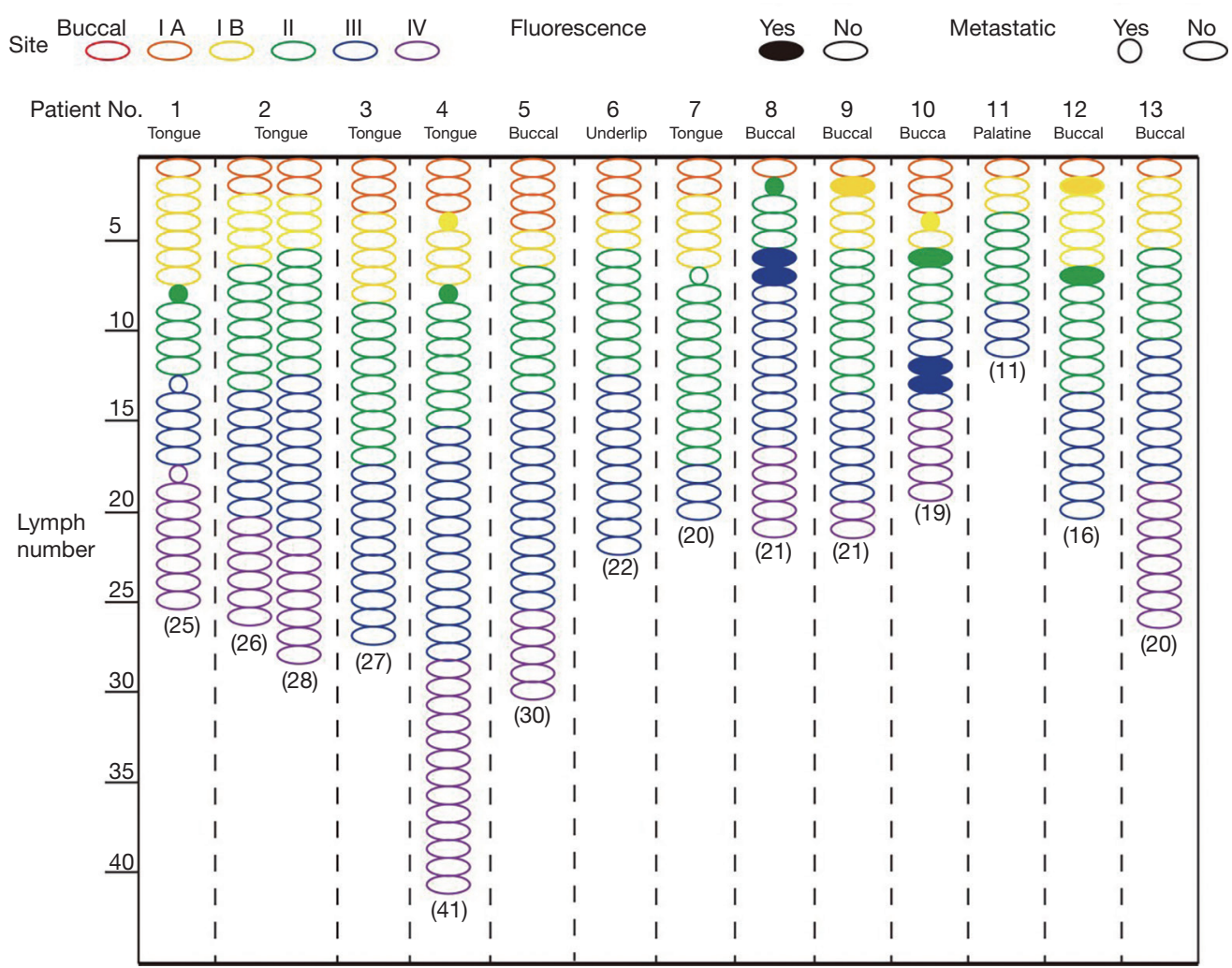

Figure 4 NIR fluorescence imaging for HNSCC patients who received intravenous injection of ICG. NIR, near-infrared; HNSCC, head and neck squamous cell carcinoma; ICG, indocyanine green.

Table 2 Diagnostic statistics for intraoperative NIR fluorescence imaging in vivo

\begin{tabular}{lcccccc}
\hline Administration method & Sensitivity (\%) & Specificity (\%) & PPV (\%) & NPV (\%) & Accuracy (\%) & $\begin{array}{c}\text { Accuracy estimate rate for } \\
\text { regional lymph nodes (\%) }\end{array}$ \\
\hline Intravenous injection of ICG & 62.5 & 98.1 & 45.5 & 99.1 & 97.3 & 92.3 \\
Peritumoral injection of ICG & 75 & 89.1 & 12.8 & 99.4 & 88.8 & 100 \\
\hline
\end{tabular}

NIR, near-infrared.

patient) LNs were sorted by region (Figure 5): 2 (0.5\%) LNs were located in the buccal region, 15 (3.9\%) LNs were located in level Ia, $69(18.0 \%)$ LNs were located in level $\mathrm{Ib}, 97$ (25.3\%) LNs were located in level II, 113 (29.4\%) LNs were located in level III, and 88 (22.9\%) LNs were located in level IV. Following postoperative paraffin section, 8 (2.1\%) metastatic LNs were identified, 6 of which (75\%) were fluorescence-positive, and 2 of which $(25 \%)$ were fluorescence-negative. With the $\mathrm{LN}$ as the unit, the sensitivity and specificity were $75 \%$ and $89.1 \%$ respectively, the PPV and NPV were $12.8 \%$ and $99.4 \%$ respectively, and the accuracy was $88.8 \%$ (Table 2). With the patient as the unit, the accuracy of NIR fluorescence imaging using peritumoral injection of ICG combined with intraoperative frozen section was $100 \%$.

\section{Fluorescence intensity quantification for ex vivo lymph nodes}

After LNs were dissected and sorted, the fluorescence intensities were measured by a hand-held NIF spectrometer. For patients who received intravenous injection of ICG, the mean fluorescence intensity (MFI) of metastatic LNs (83.5 \pm 36.5 , range, $37-135)$ was significantly higher than that 


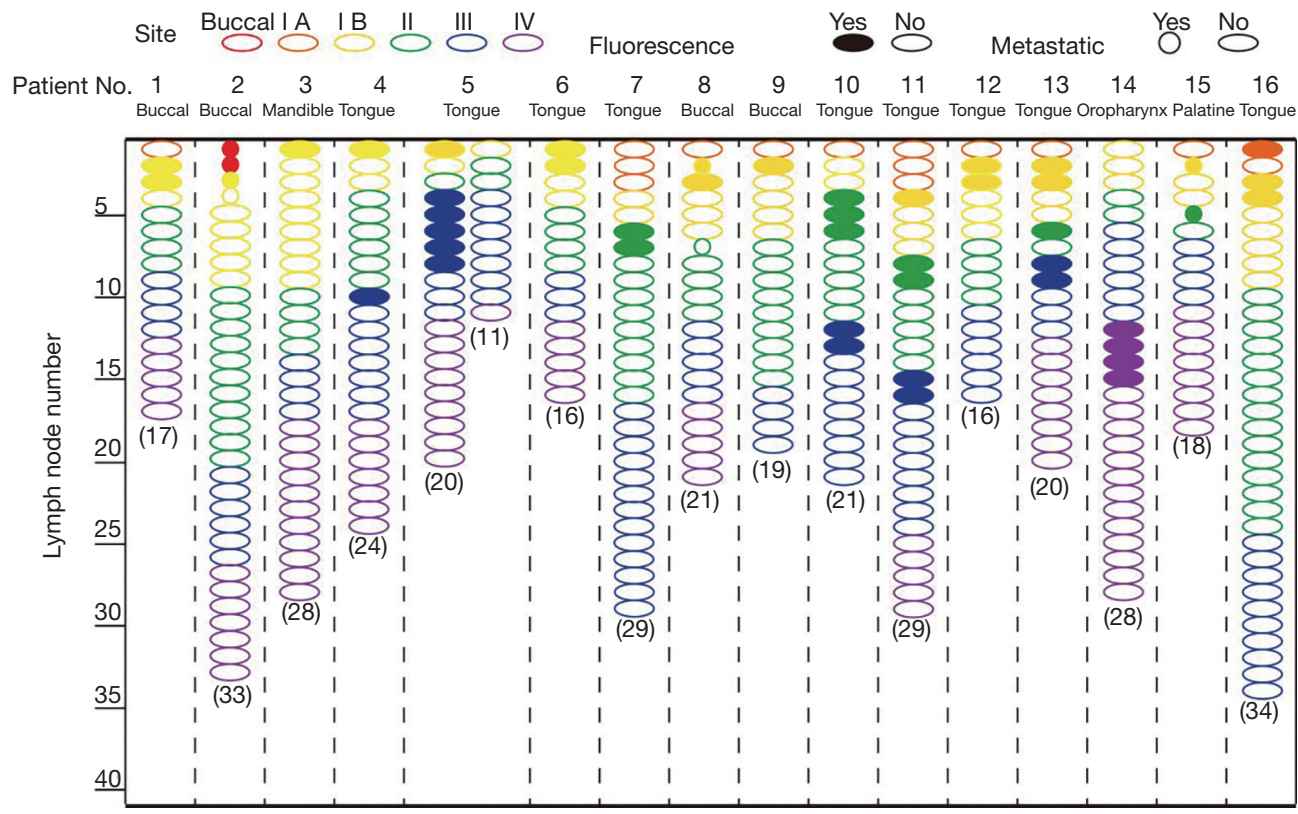

Figure 5 NIR fluorescence imaging for HNSCC patients who received peritumoral injection of ICG. NIR, near-infrared; HNSCC, head and neck squamous cell carcinoma; LNs, lymph nodes; ICG, indocyanine green.

of non-metastatic LNs $(32.0 \pm 15.2$, range, 11-135) $(\mathrm{P}<0.001)$ (Figure 6A). For patients who received peritumoral injection of ICG, the MFI of metastatic LNs (146.8 \pm 46.9 , range 46-214) was also higher than that of non-metastatic LNs $(80.3 \pm 73.3$, range $1-275)(\mathrm{P}<0.05)$ (Figure $6 A)$. Moreover, the MFI of LNs from patients who received peritumoral injection of ICG $(81.7 \pm 73.5)$ was higher than that of patients who received intravenous injection of ICG $(33.2 \pm 17.9)(\mathrm{P}<0.001)$ (Figure 6B).

To explore the feasibility of estimating the status of LNs by fluorescence intensity measuring, the relationship between fluorescence intensity and metastatic status were further analyzed. For patients who received intravenous injection of ICG, the optimal threshold value for MFI was found to be 81.5 at which a $62.5 \%$ sensitivity and a $99.39 \%$ specificity were reached (LR 101.9). The PPV and NPV were $71.4 \%$ and $99.1 \%$ respectively (Table 3 ). For patients who received peritumoral injection of ICG, the optimal threshold value was 118.5 at which an $87.5 \%$ sensitivity and a $73.67 \%$ specificity were reached (LR 3.3), with a PPV and NPV of $6.6 \%$ and $99.6 \%$ respectively (Table 3). ROC curve analysis showed the AUC obtained for intravenous injection of ICG was 0.91 [95\% confidence interval (CI) 0.83-0.99], and the AUC for peritumoral injection of ICG was 0.78 (95\% CI 0.68-0.87) suggesting that intravenous ICG administration is more valuable in determining $\mathrm{LN}$ status $(\mathrm{P}<0.001)$ (Figure 6C).

To identify the minimum number of LNs required for pathological examination to achieve accurate evaluation of regional LN status, we ranked the LNs according to the fluorescence intensity in each patient (Figure 7). For patients who received intravenous injection of ICG, the first 6 highest LNs in fluorescence intensity needed to undergo pathological examination to ensure the accurate evaluation of regional LN status (specificity 78.4\%, sensitivity $100 \%$ ), while only the first 4 highest LNs in fluorescence intensity were needed for patients who received peritumoral injection of ICG (specificity $84.8 \%$, sensitivity $87.5 \%$ ) (Figure $8 A$ ). For individual metastatic LNs, the data suggest that the optimal threshold for detection number was six for intravenous injection of ICG with a specificity of $78.4 \%$ and a sensitivity of $100 \%$; peritumoral injection of ICG also yielded an optimal threshold of six, with a specificity of $76.6 \%$ and a sensitivity of $100 \%$ (Figure $8 B$ and Table 4).

\section{Discussion}

ICG-based NIR fluorescence imaging has already been introduced in head and neck oncology to assist with the resection of primary tumor tissues and map SLNs. In our 

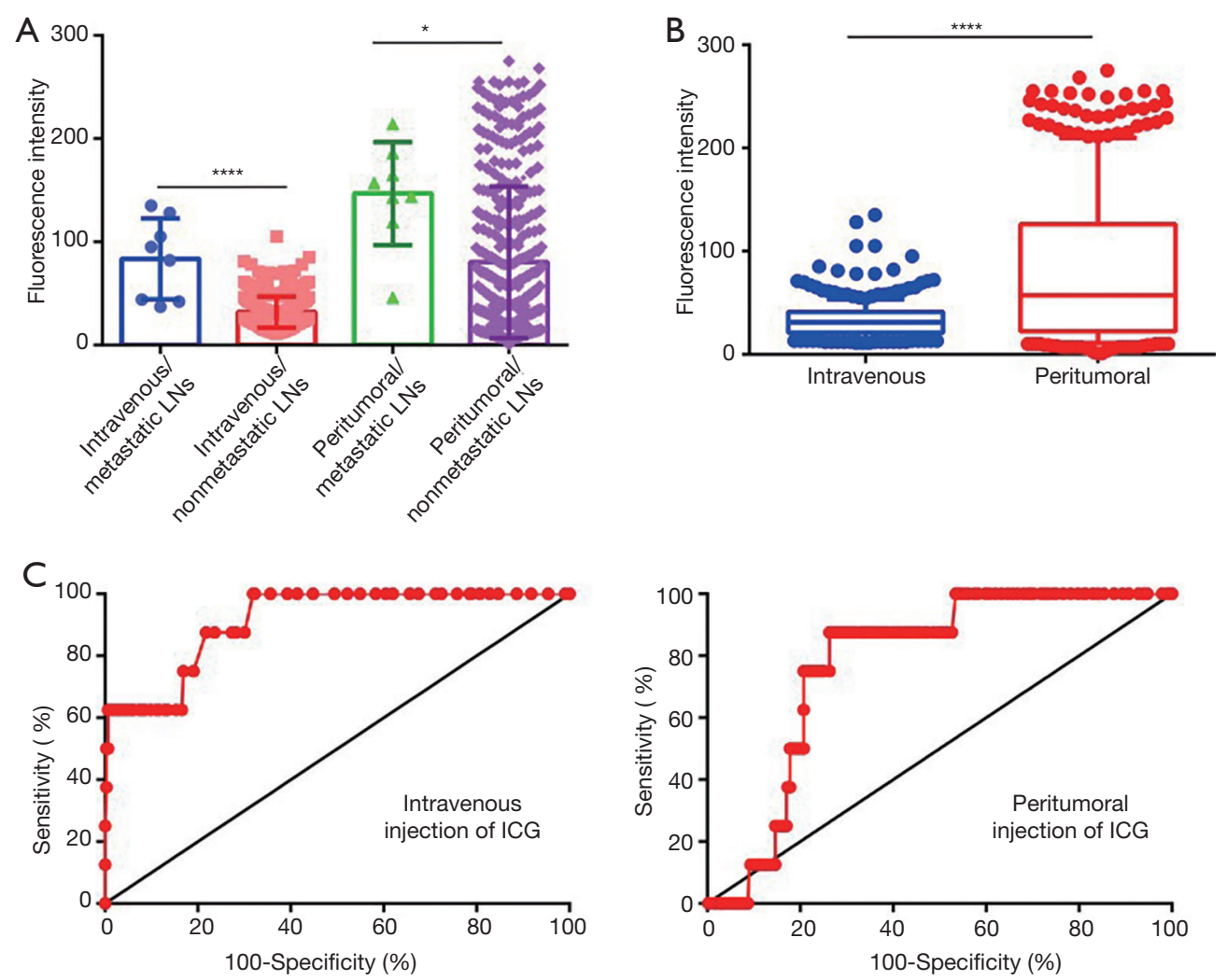

Figure 6 Diagnostic value comparison between intravenous injection of ICG and peritumoral injection of ICG. (A) The fluorescence intensity of metastatic or non-metastatic LNs ex vivo after intravenous injection of ICG and peritumoral injection of ICG. (B) The fluorescence intensity distribution of LNs ex vivo after intravenous injection of ICG and peritumoral injection of ICG. (C) ROC curve evaluation showed that intravenous ICG administration (0.91, 95\% CI: $0.83-0.99)$ is more valuable than peritumoral administration in determining LN status (0.78, 95\% CI: 0.68-0.87). *, $\mathrm{P}<0.05$; ***, $\mathrm{P}<0.0001$. ICG, indocyanine green; LNs, lymph nodes.

Table 3 Diagnostic statistics for fluorescence intensity quantification of lymph nodes ex vivo

\begin{tabular}{lcccccc}
\hline Threshold values & Sensitivity (\%) & Specificity (\%) & PPV (\%) & NPV (\%) & Accuracy (\%) & Preselected number of LNs (\%) \\
\hline Intravenous (MFI >81.5) & 62.5 & 99.39 & 71.4 & 99.1 & 98.5 & $7(2.1)$ \\
Peritumoral (MFI >118.5) & 87.5 & 73.67 & 6.6 & 99.6 & 74.0 & $106(27.6)$ \\
\hline
\end{tabular}

previous studies, we also confirmed the value of ICG-based NIR fluorescence imaging in the delineation of the tumor boundary and the identification of residual tumor cells (26,27). Even more surprising, we have further observed that ICG fluorescence positive-LNs were associated with metastasis. The data showed that based on the NIR imaging results in vivo, the sensitivity and specificity of evaluating LN status were $62.5 \%$ and $98.1 \%$ respectively, and the accuracy reached $97.3 \%$. Moreover, combined with the intraoperative frozen section, the $\mathrm{LN}$ status of the neck was accurately identified in 12 patients (92.3\%).
This proves that intravenous injection of ICG combined with intraoperative frozen section is an effective method for accurately evaluating the status of regional LNs of HNSCC patients intraoperatively. In a review of the relevant literature, we found that only two research groups have used intravenous injection of NIR fluorescence agents [human serum albumin (HSA)-ICG and panitumumabIRDye800CW] to evaluate the status of LNs ex vivo in patients with HNSCC $(23,28)$. Among these, Digonnet et al. discovered that after intravenous injection of HSA-ICG, the sensitivity and specificity for identifying 


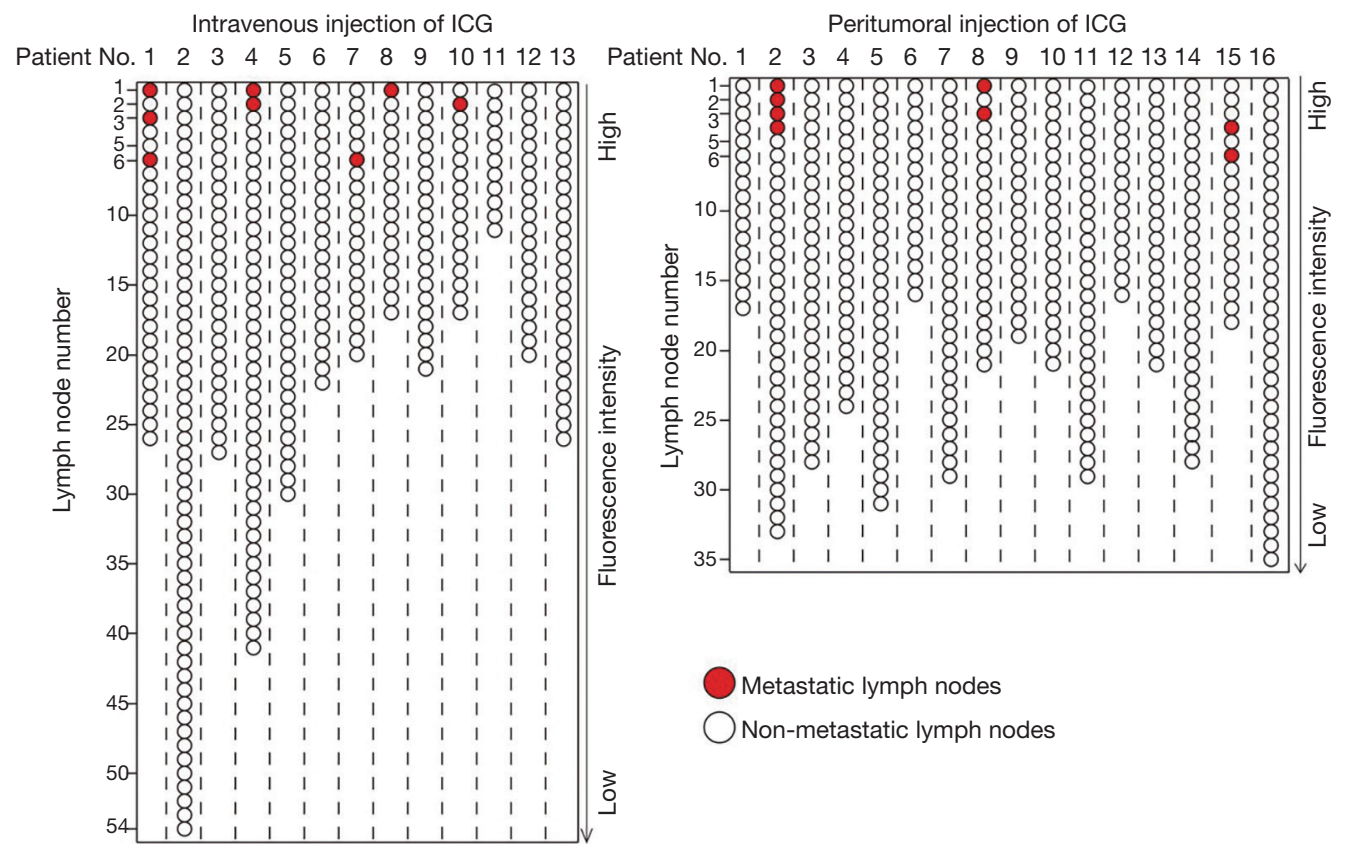

Figure 7 Fluorescence intensity-based ranking of LNs from patients who received intravenous injection of ICG and peritumoral injection of ICG. LNs, lymph nodes; ICG, indocyanine green.

metastatic LNs were $76.5 \%$ and $76.7 \%$ respectively. Meanwhile, Nishio et al. found that after intravenous injection of panitumumab-IRDye $800 \mathrm{CW}$, the sensitivity and specificity were $84.6 \%$ and $94 \%$ respectively (23). In the present study, we found that after intravenous injection of ICG, the sensitivity and specificity were $62.5 \%$ and $99.39 \%$, respectively. These results indicate to us that although intravenous injection of untargeted fluorescence agents like ICG can provide new insight into evaluating LN status, fluorescence agents coupled with tumortargeted protein may further improve the diagnostic accuracy.

SLNB has already been widely introduced to the management of HNSCC patients with T1-T2 cN0 status using methylene blue, radiocolloid, or ICG (29). Compared to methylene blue and radiocolloid, ICG-based SLNB has been proven to improve the detection rate of SLN, especially for tumors in the floor of the mouth (30). In this study, SLNs could be detected in all patients (16/16), which is in accordance with other studies $(25,31,32)$. After further consulting the literature, we found that the sensitivity and NPV in different studies showed obvious differences in the detection of metastatic LNs. For example, in the study by Peng et al., the sensitivity and NPV both reached up to $100 \%$ (25), while in Al-Dam et al.'s study, false negatives appeared in half of the clinical $\mathrm{LN}$-positive $(\mathrm{cN}+)$ patients (32). Meanwhile, in the present study, the sensitivity and NPV were $75 \%$ and $99.4 \%$ respectively, while no false negatives occurred in $\mathrm{cN}+$ patients. We speculate that the difference may be related to the ICG dosages administered and the detection sensitivity of the equipment. Moreover, in view of the small sample included in this study, we believe more patients need to be enrolled to further verify the value of ICG-based SLNB in the future.

Unlike the SLNB that uses peritumoral injection of ICG, reports on the use of intravenous injection of ICG to locate the at-risk $\mathrm{LNs}$ in the neck region are lacking. The value of this method still needs to be confirmed, and its relative merit compared to SLNB using peritumoral injection of ICG also needs to be assessed. In this study, we systematically compared the differences of these two methods in locating the metastatic LNs of HNSCC patients. During the surgery, SLNB using peritumoral injection of ICG could detect more fluorescence-positive LNs (average number of LNs: 2.94). Although the sensitivity improved, the specificity decreased, and the pathological workload also increased. For intravenous injection of ICG, fewer fluorescence-positive LNs (average number of LNs: 0.85 ) were detected. Despite the increased 

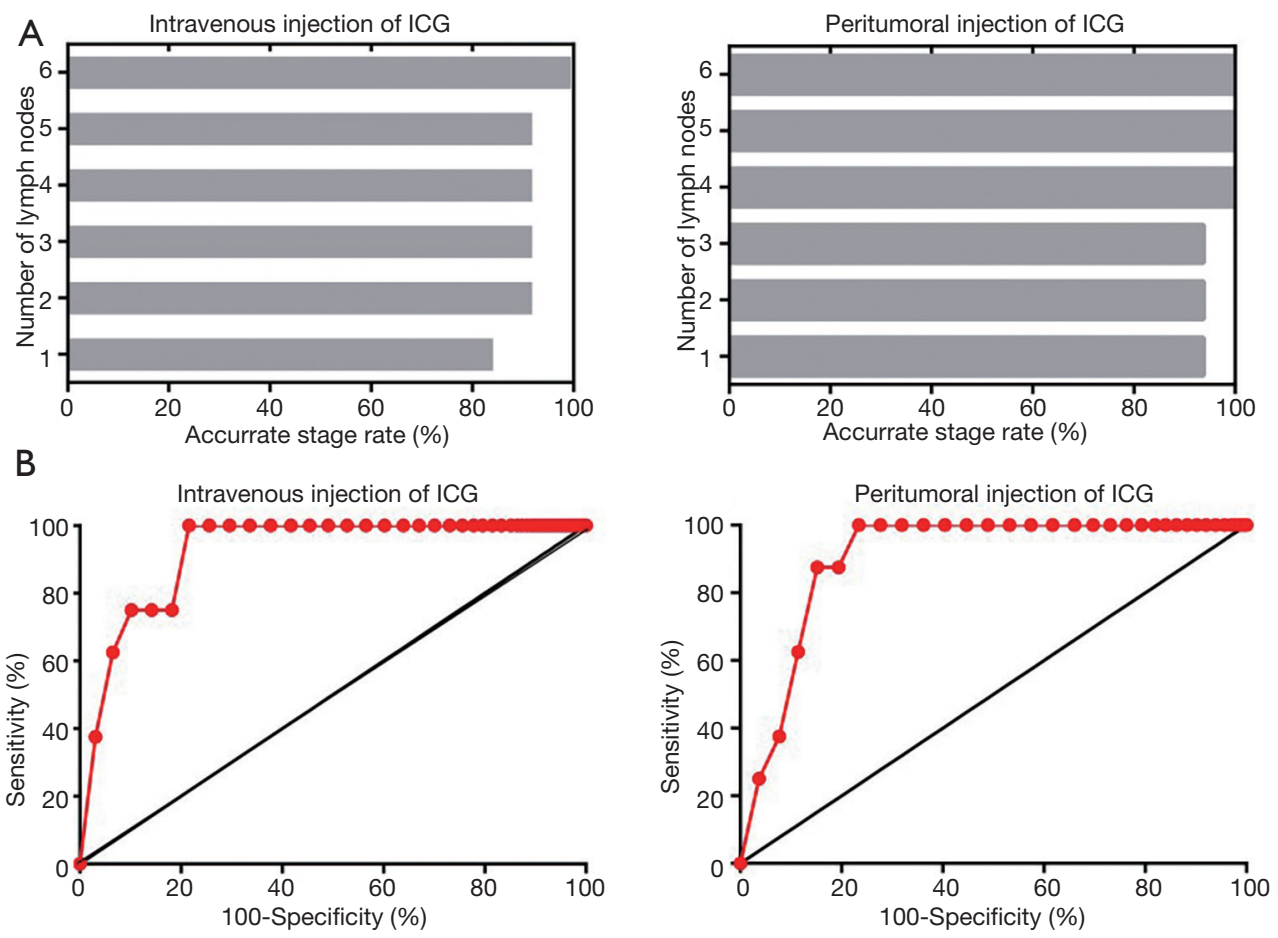

Figure 8 The minimum number of LNs required for pathological examination to achieve accurate evaluation of regional LN status. (A) Detection of the first $6 \mathrm{LNs}$ with the highest fluorescence intensity could ensure the accurate evaluation of cervical LN status after intravenous injection of ICG while the first 4 LNs with the highest fluorescence intensity could ensure the accurate evaluation after peritumoral injection of ICG. (B) Sensitivity and specificity based on the number of LNs per patient that needed to be assessed by pathology. LNs, lymph nodes; ICG, indocyanine green.

Table 4 Diagnostic statistics for detecting the number of LNs ex vivo

\begin{tabular}{lccccc}
\hline Threshold values & Sensitivity (\%) & Specificity (\%) & PPV (\%) & NPV (\%) & Accuracy (\%) \\
\hline Intravenous (lymph node number =6) & 100 & 78.4 & 10.3 & 100 & 78.9 \\
Peritumoral (lymph node number =6) & 100 & 76.6 & 8.3 & 100 & 77.1 \\
\hline
\end{tabular}

LNs, lymph nodes.

specificity and the reduced pathological workload, there is also a risk of missed diagnosis (1/13). After the surgery, fluorescence intensity quantitative analysis was also performed. The results showed that ICG administration intravenously was more valuable in determining the $\mathrm{LN}$ status [area under the curve (AUC): 0.91 vs. $0.78, \mathrm{P}<0.001$ ]. Moreover, the pathological workload was also significantly relieved through the use of intravenous injection of ICG (average number of LNS requiring frozen section: 0.54 vs. 6.63). Furthermore, ICG administration intravenously could also aid in the resection of the primary tumor beside the location of metastatic LNs. In view of this, we recommend ICG administration intravenously for locating metastatic LNs.

In conventional clinical work, pathological examination needs to be performed in the LNs dissected, which greatly increases the burden to the pathologists and patients. In this study, we demonstrated that preselecting at-risk LNs with NIR fluorescence imaging could greatly reduce the number of LNs requiring detection. After the ranking of LNs according to the fluorescence intensity, the number of LNs required for pathological examination to guarantee the accurate staging of cervical LN status was 4 and 6 for intravenous ICG injection and peritumoral ICG injection 
respectively. If this method could be further verified with a large sample, about $80 \%$ of LNs could be spared of further pathological examination.

\section{Conclusions}

The accurate evaluation of cervical LN status is essential for the diagnosis and treatment of HNSCC patients. In this study, we demonstrated the application value of NIR fluorescence imaging using ICG in the preselection of atrisk LNs in vivo and ex vivo. With the assistance of NIR fluorescence imaging using ICG, both the postoperative complications of patients and the workload of pathologists will be reduced.

\section{Acknowledgments}

The authors appreciate the academic support from AME Head and Neck Cancer Collaborative Group.

Funding: This work was supported by Nanjing Clinical Research Center for Oral Diseases (2019060009); the Jiangsu Province Science \& Technology Department (BE2018618); and the State Commission of Science \& Technology of China (2016YFC0104100).

\section{Footnote}

Reporting Checklist: The authors have completed the STARD reporting checklist. Available at http://dx.doi.org/10.21037/ atm-21-392

Data Sharing Statement: Available at http://dx.doi. org/10.21037/atm-21-392

Conflicts of Interest: All authors have completed the ICMJE uniform disclosure form (available at http://dx.doi. org/10.21037/atm-21-392). Dr. Hu reports grants from Nanjing Clinical Research Center for Oral Diseases, during the conduct of the study; Dr. Cai reports grants from the State Commission of Science \& Technology of China, during the conduct of the study; Dr. Wang reports grants from the Jiangsu Province Science \& Technology Department, grants from Nanjing Clinical Research Center for Oral Diseases, during the conduct of the study. The other authors have no conflicts of interest to declare.

Ethical Statement: The authors are accountable for all aspects of the work in ensuring that questions related to the accuracy or integrity of any part of the work are appropriately investigated and resolved. The study was conducted in accordance with the Declaration of Helsinki (as revised in 2013). The study was approved by the medical ethics committee of the Institute Affiliated Stomatology Hospital, Medical School of Nanjing University (2017NL001) and informed consent was taken from all individual participants.

Open Access Statement: This is an Open Access article distributed in accordance with the Creative Commons Attribution-NonCommercial-NoDerivs 4.0 International License (CC BY-NC-ND 4.0), which permits the noncommercial replication and distribution of the article with the strict proviso that no changes or edits are made and the original work is properly cited (including links to both the formal publication through the relevant DOI and the license). See: https://creativecommons.org/licenses/by-nc-nd/4.0/.

\section{References}

1. Mes SW, Leemans CR, Brakenhoff RH. Applications of molecular diagnostics for personalized treatment of head and neck cancer: state of the art. Expert Rev Mol Diagn 2016;16:205-21.

2. Noorlag R, van Kempen P, Stegeman I, et al. The diagnostic value of $11 \mathrm{q} 13$ amplification and protein expression in the detection of nodal metastasis from oral squamous cell carcinoma: a systematic review and metaanalysis. Virchows Archiv 2015;466:363-73.

3. Brennan PA, Subramaniam S, Tsioryannis C, et al. An update on the latest evidence for managing the clinically negative neck ( $\mathrm{cN} 0)$ in oral squamous cell carcinoma. Oral Dis 2017;23:287-91.

4. Hanai N, Asakage T, Kiyota N, et al. Controversies in relation to neck management in N0 early oral tongue cancer. Jpn J Clin Oncol 2019;49:297-305.

5. Cui Z, Gao Y, Wang W, et al. Evaluation of Neck Lymph Node Metastasis on ContrastEnhanced Ultrasound: An Animal Study. Clin Exp Otorhinolaryngol 2017;10:109-14.

6. Salazar-Fernandez CI, Gallanaalvarez S, Pereira $\mathrm{S}$, et al. Sentinel Lymph Node Biopsy in Oral and Oropharyngeal Squamous Cell Carcinoma: Statistical Validation and Impact of Micrometastasis Involvement on the Neck Dissection Decision. J Oral Maxillofac Surg 
2015;73:1403-9.

7. Ishida T, Hijioka H, Kume K, et al. A diagnosis system for detecting cervical lymph node metastasis in oral squamous cell carcinoma: Collective consideration of the results of multiple imaging modalities. Journal of Oral and Maxillofacial Surgery, Medicine, and Pathology 2017;29:210-6.

8. Rammeh S, Romdhane E, Sassi A, et al. Accuracy of fine-needle aspiration cytology of head and neck masses. Diagn Cytopathol 2019;47:394-9.

9. Caudle AS, Kuerer HM, Krishnamurthy S, et al. Feasibility of fine-needle aspiration for assessing responses to chemotherapy in metastatic nodes marked with clips in breast cancer: A prospective registry study. Cancer 2019;125:365-73.

10. Alitalo K, Tammela T, Petrova TV. Lymphangiogenesis in development and human disease. Nature 2005;438:946-53.

11. Civantos FJ, Stoeckli SJ, Takes RP, et al. What is the role of sentinel lymph node biopsy in the management of oral cancer in 2010? Eur Arch Otorhinolaryngol 2010;267:839-44.

12. Qiu SQ, Zhang GJ, Jansen L, et al. Evolution in sentinel lymph node biopsy in breast cancer. Crit Rev Oncol Hematol 2018;123:83-94.

13. Zeng HC, Hu JL, Bai JW, et al. Detection of Sentinel Lymph Nodes with Near-Infrared Imaging in Malignancies. Mol Imaging Biol 2019;21:219-27.

14. Vishnoi JR, Kumar V, Gupta S, et al. Outcome of sentinel lymph node biopsy in early-stage squamous cell carcinoma of the oral cavity with methylene blue dye alone: a prospective validation study. Br J Oral Maxillofac Surg 2019;57:755-9.

15. Li J, Lu M, Cheng X, et al. How Pre-operative Sentinel Lymph Node Contrast-Enhanced Ultrasound Helps Intra-operative Sentinel Lymph Node Biopsy in Breast Cancer: Initial Experience. Ultrasound Med Biol 2019;45:1865-73.

16. Nielsen Moody A, Bull J, Culpan AM, et al. Preoperative sentinel lymph node identification, biopsy and localisation using contrast enhanced ultrasound (CEUS) in patients with breast cancer: a systematic review and meta-analysis. Clin Radiol 2017;72:959-71.

17. Hikage $M$, Gonda K, Takeda M, et al. Nano-imaging of the lymph network structure with quantum dots. Nanotechnology 2010;21:185103.

18. Nakahara T, Kitagawa Y, Yakeuchi H, et al. Preoperative lymphoscintigraphy for detection of sentinel lymph node in patients with gastric cancer--initial experience. Ann Surg Oncol 2008;15:1447-53.

19. Gibbs SL. Near infrared fluorescence for image-guided surgery. Quant Imaging Med Surg 2012;2:177-87.

20. Keereweer S, Van Driel PB, Snoeks TJ, et al. Optical image-guided cancer surgery: challenges and limitations. Clin Cancer Res 2013;19:3745-54.

21. Zhang RR, Schroeder AB, Grudzinski JJ, et al. Beyond the margins: real-time detection of cancer using targeted fluorophores. Nat Rev Clin Oncol 2017;14:347-64.

22. Hong G, Antaris AL, Dai H. Near-infrared fluorophores for biomedical imaging. Nature Biomedical Engineering 2017;1.

23. Nishio N, van den Berg N, van Keulen S, et al. Optical molecular imaging can differentiate metastatic from benign lymph nodes in head and neck cancer. Nat Commun 2019;10:5044.

24. Cuccurullo V, Mansi L. AJCC Cancer Staging Handbook: from the AJCC Cancer Staging Manual (7th edition). Eur J Nucl Med Mol Imaging 2011;38:408.

25. Peng H, Wang SJ, Niu X, et al. Sentinel node biopsy using indocyanine green in oral/oropharyngeal cancer. World J Surg Oncol 2015;13:278.

26. Wang Y, Xie D, Wang Z, et al. Kinetics of indocyanine green: Optimizing tumor to normal tissue fluorescence in image-guided oral cancer surgery applications. Head Neck 2019;41:1032-8.

27. Pan J, Deng H, Hu S, et al. Real-time surveillance of surgical margins via ICG-based near-infrared fluorescence imaging in patients with OSCC. World J Surg Oncol 2020;18:96.

28. Digonnet A, van Kerckhove S, Moreau M, et al. Near infrared fluorescent imaging after intravenous injection of indocyanine green during neck dissection in patients with head and neck cancer: A feasibility study. Head Neck 2016;38 Suppl 1:E1833-7.

29. Green B, Blythe J, Brennan PA. Sentinel lymph node biopsy for head and neck mucosal cancers - an update on the current evidence. Oral Dis 2016;22:498-502.

30. van den Berg NS, Brouwer OR, Klop WM, et al. Concomitant radio- and fluorescence-guided sentinel lymph node biopsy in squamous cell carcinoma of the oral cavity using ICG-(99m)Tc-nanocolloid. Eur J Nucl Med Mol Imaging 2012;39:1128-36.

31. Bredell MG. Sentinel lymph node mapping by indocyanine green fluorescence imaging in oropharyngeal cancer - preliminary experience. Head Neck Oncol 2010;2:31. 
32. Al-Dam A, Precht C, Barbe A, et al. Sensitivity and specificity of sentinel lymph node biopsy in patients with oral squamous cell carcinomas using indocyanine green fluorescence imaging. J Craniomaxillofac Surg

Cite this article as: Xia C, Zhou Q, Zhang Q, Hu S, Meacci E, Matsuura Y, Durand M, Hu Q, Cai H, Wang Y. Comparative study on the diagnostic value of intravenous/ peritumoral injection of indocyanine green for metastatic lymph node location in patients with head and neck squamous cell carcinoma (HNSCC). Ann Transl Med 2021;9(6):507. doi: 10.21037/atm-21-392
2018;46:1379-84.

(English Language Editor: J. Gray) 